\title{
The natural course of myasthenia gravis
}

\author{
The outlook has improved substantially
}

The natural history of myasthenia gravis is uncertain. Anticholinesterase preparations were introduced in $1934,{ }^{1}$ and we now accept a therapeutic response to these as part of the definition of the disease. Historical evidence of outcome is unreliable as in the early series before the advent of thymectomy the less severe forms of the disease were almost certainly missed. The combination of electromyography, single fibre electromyography, and the measurement of acetylcholine receptor antibodies now allows the detection of milder cases and the exclusion of other myasthenic syndromes. Yet despite advances in our understanding of the pathophysiology of myasthenia gravis its treatment is still controversial-because of the lack of data from controlled clinical trials. ${ }^{2}$ Such trials are difficult to carry out because of the long duration of treatment required before benefit may be obtained and the fluctuations in the natural course of the disease. Indeed these trials may now be unethical as large uncontrolled studies have shown the beneficial effects of the various treatments used.

A recent study by Oosterhuis is of interest. ${ }^{3} \mathrm{He}$ has reviewed retrospectively all patients diagnosed as having myasthenia gravis at the university department of neurology in Amsterdam from 1926 to 1965 . Thymectomy was not used before 1965 for patients who did not have a thymoma. If we assume that anticholinesterase drugs do not influence the outcome of the disease then the outcome in the early patients reflects the natural course of myasthenia gravis. A quarter of the patients had died by 1965 . In $1985,22 \%$ of the original cohort were in complete remission, $34 \%$ had improved, $16 \%$ were unchanged, and $3 \%$ had deteriorated.

In another series of patients with myasthenia-those treated at the National Hospital, Queen's Square, without surgery between 1934 and 1956-29\% died of myasthenia and $4 \cdot 5 \%$ of other causes. ${ }^{+}$Most of the deaths occurred two to six years after onset of symptoms regardless of whether the patients had a thymoma or not. After thymectomy was introduced the mortality among those without a tumour was halved. It is interesting that these results were achieved before an immunological role for the thymus was established, as it was not until 1961 that Miller's experiments led to an appreciation of the true function of the thymus. ${ }^{5}$ Similarly, in the United States before thymectomy only $39 \%$ of patients were reported to improve and the mortality was $30 \% .^{6}$

In the patients described by Oosterhuis the initial signs were ocular in over a half of the cases; bulbar weakness was the next most common presentation, followed by weakness of the limbs. ${ }^{3}$ In one third of the patients with ocular symptoms these remained the only feature, while the remainder developed generalised disease, usually within two years. Spontaneous remissions in the first year of disease occurred in $22 \%$ of patients: half of these relapsed after three to 12 months and the other half after between one and six years.

These statistics may be compared with a larger series of patients studied between 1965 and 1984, in which complete remission occurred in $37 \%$ and death in $9 \% .^{7}$ Death was due to myasthenia itself in $5 \cdot 2 \%$, to side effects of immunosuppressive drugs in $1 \cdot 8 \%$, and to invasiveness of the tumour in $2 \%$.

Thymectomy was first undertaken in Britain in 1942, and Simpson's early studies showed that the operation benefited most patients but especially those without a thymoma. ${ }^{4}$ Furthermore, the best results occurred after early operation. These results allowed the formulation of a treatment policy, and Simpson and Thomaides have subsequently reported virtual recovery in more than $90 \%$ of cases. ${ }^{8} \mathrm{~A}$ retrospective comparison of medical treatment (without corticosteroids) with surgical treatment showed that patients treated by thymectomy were more likely to achieve remission and less likely to die of their disease. ${ }^{9}$ Survival was also substantially improved by the recognition of cholinergic crises and by the introduction of positive pressure ventilation in 1953. Although thymectomy is less effective in patients with a thymoma it should still be undertaken to decrease the risk of local invasion by the tumour.

As myasthenia gravis is well established to be an autoimmune disorder, what is the therapeutic contribution of immunosuppression? Corticosteroids and plasma exchange seem to be particularly useful in patients who are too weak to undergo thymectomy and in those who remain severely disabled after thymectomy. Corticosteroids induce remission too early, however, for this to be the result of immunosuppression; they probably act by protecting the acetylcholine receptor from immunological attack. Corticosteroids may be expected to cause remission in $80 \%$ of patients, ${ }^{10}$ and the remission can be maintained by relatively small doses given on alternate days. ${ }^{11}$

Azathioprine has been used in the treatment of myasthenia for 20 years. It takes around seven months to achieve remission. ${ }^{12}$ In the only controlled trial of immunosuppression cyclosporin A was shown to produce a significant improvement in muscle strength but was associated with sufficient toxicity to preclude its use as a first line immunosuppressive 
drug. ${ }^{13}$ A recent review of experience with azathioprine in 27 patients over a median duration of seven years suggested that side effects were few, and treatment had had to be discontinued in only four patients ${ }^{14}$; all of the others improved and were able to take lower dosages of anticholinesterase drugs and corticosteroids. It has been argued that corticosteroids and immunosuppressants do not diminish the already low mortality from myasthenia gravis, ${ }^{8}$ but there is little doubt that they improve the quality of life. ${ }^{14}$

Despite the lack of evidence from rigorous controlled trials the conclusion must be that the outlook for patients with myasthenia gravis has improved considerably over the past

Walker M. Treatment of myasthenia gravis with physostigmine. Lancet 1934;i: 1200-1.

Rowland LP. Controversies about the treatment of myasthenia gravis. 7 Neurol Neurosurg Psychiatry 1980;43:644-59.

3 Oosterhuis HJGH. The natural course of myasthenia gravis: a long term follow up study. F Neurol Neurosurg Psychiatry 1989;52:1121-7.

Simpson JA. Evaluation of thymectomy in myasthenia gravis. Brain 1958;81:112-45.

5 Miller JFAP. Immunological function of the thymus. Lancet 1961;ii:748-9.

6 Grob D, Brunner NG, Namba T. The natural course of myasthenia gravis and effects of various therapeutic measures. Ann NY Acad Sci 1981;377:652-70.

7 Oosterhuis $\mathrm{HJGH}$. Long term effects of treatment in 374 patients with myasthenia gravis. In: de Baets M, Oosterhuis HJGH, Toyka K, eds. Monographs in allergy. Vol 25. Basle: Karger, 1988:75-85.

8 Simpson JA, Thomaides T. Treatment of myasthenia gravis: an audit. $O \mathcal{F}$ Med 1987;64:693-704 decade. The judicious use of thymectomy, corticosteroids, and, if necessary, the more powerful immunosuppressive drugs offers them a combination of reduced morbidity and a high rate of remission. The mortality from myasthenia gravis in the 1990s should be negligible. We agree with Rowland: the prognosis in myasthenia has improved so much we must be doing something right.

V FONSECA Senior Registrar

C W H HAVARI) Consultant Physician and Endocrinologist

Royal Free Hospital, London NW3 2QG

9 Buckingham JM, Howard FM, Bernatz PE, et al. The value of thymectomy in myasthenia gravis: computer-assisted matched study. Ann Surg 1976;184:453-5.

10 Pascuzzi RM Coslett HB, Johns TR. Long-term corticosteroid treatment of myasthenia gravis: report of 116 patients. Ann Neurol 1984;15:291-8.

11 Havard CWH, Fonseca V. New treatment approaches to myasthenia gravis. Drugs 1990;39:66-73.

12 Mertens HG, Hertel G, Reuther P, Ricker K. Effect of immunosuppressive drugs (azathioprine) Ann NY Acad Sci 1981;377:691-9.

13 Tindall RSA, Rollins JA, Phillips JT, Greenlee RG, Wells L, Belenduick G. Preliminary results of a double-blind, randomised, placebo-controlled trial of cyclosporin in myasthenia gravis. $N$ Engl 7 Med 1987;316:719-24

14 Fonseca $\mathrm{V}$, Havard $\mathrm{CWH}$. Long term treatment of myasthenia gravis with azathioprine. Postgrad Med f 1990;66:102-5.

\title{
Human Fertilisation and Embryology Bill goes to report stage
}

\author{
Donor anonymity to go? Is GIFT included?
}

The Human Fertilisation and Embryology Bill has now completed its committee stage and will shortly return to the House of Commons for report. It has come out of committee containing two major ambiguities. These must be resolved before the bill becomes law.

\section{Donor anonymity}

The first concerns anonymity for semen donors. Nearly half of all infertility is caused by problems in the man, notably deficient concentration and quality of spermatozoa or total lack of spermatozoa.' Our limited understanding about the factors controlling spermatogenesis means that there are no reliable medical or surgical treatments for these conditions. For a small number of couples sufficient motile sperm may be recovered from the ejaculate to allow an egg to be inseminated in vitro. The quality of the sperm may, however, be so poor that fertilisation does not occur. For these unfortunate couples, and for those couples in which the man is azoospermic, the only treatment by which a pregnancy can be achieved is that by donor insemination.

Spermatozoa are donated anonymously for insemination by fertile men who wish to help infertile couples to have a child. Clinics offering donor insemination take great care to match the physical characteristics of the infertile male partner to those of the donor and to screen potential donors for sexually transmissible agents (including HIV) and genetically carried disease $^{2-4}$ The anonymity of these individual donors has always been carefully protected. The Human Fertilisation and Embryology Bill requires all donor insemination centres to be licensed and to store in the files of the newly created licensing authority identifying information about any person whose gametes are used for any assisted conception procedure including donor insemination. Failure to comply constitutes a breach of the criminal law. The intentions of this proposed regulation are clear and admirable and follow recommendations in the Warnock report. ${ }^{5}$ Firstly, it will enable standards of practice, including counselling, to be set and monitored and accurate statistics about donor insemination in this country to be collected. ${ }^{+}$Secondly, if a child born after donor insemination should be found to have a genetic disease then insemination of other patients using the same sperm may be prevented, and the donor can be traced and advised of the risk that he might have of passing on a genetic disease to his own offspring. Indeed, clinics practising donor insemination already keep detailed and totally confidential records of births and donors for this specific eventuality. But the bill also allows for a new practice. A child born after donor insemination on reaching the age of 18 years may request information about his or her genetic origins. Despite assurances by the minister in committee that the intention of the law was to provide only non-identifying information to the child-as was recommended by the Warnock committee and in the government white paper-an amendment to include the wording "non-identifying" was defeated in committee. ${ }^{6}$ Thus the law as it now stands does not preclude identifying information about the donor being released by the licensing authority.

Opinions are polarised as to whether this new lack of guaranteed anonymity for donors is a good or a bad thing. ${ }^{78}$ On the one hand there is a body of opinion that holds strongly that it is in the child's interests to have access to its genetic father's identity should he or she wish to. This interest is held to supersede the wishes of the donor to remain anonymous. This may be a strongly held belief but there are very few data to support it. A comparison is often made with adoption, for which the law was changed recently to allow children who have been adopted to seek out the woman who gave birth to them - their genetic mother. ${ }^{9}$ This does not, however, by right enable the child to seek its genetic father, and this 